\title{
Study Questionnaire
}

1. SRF ID :

2. NAME :

3. AGE :

4. GENDER: Male ( ) Female ( )

5. CASE: ( ) CONTROL ( )

6. Designation :
A. Faculty ( )
B. Resident ( )
C. Intern ( )
D. UG Student ( )
E. Nursing Officer ( )
F. Other Nursing Staff ( )
G. AYUSH ( )
H. Supportive Staff ( )
I. Other ( )

7. Household- Currently Staying With?
A. Alone/Hostel ( )
B. Nuclear Family ( )
C. Extended/Joint Family ( )
D. Friends/Roommate ( )

8.COVID-19 RTPCR Status: have you ever tested positive for COVID-19? Yes ( ) no ( )

10. Did you have COVID related duty? YES ( ) NO ( )

11.Duration of duty (no. Of days):

12.Number of times posted (no. Of rotations):

13.Did you have any symptoms? YES ( ) NO ( )

14. If yes, what were your symptoms?
A. Fever ( )
B. Cold ( )
C. Cough ( )
D. Sore Throat ( )
E. Myalgia ( )
F. Diarrhoea ( )
G. Headache ( )
H. Breathlessness ( )
I. Chest Pain ( )
J. Loss Of Taste/Smell ( )
K. Weakness ( )
L. Skin Rash ( )
M. Conjunctivitis ( )
N. Discolouration Of Toes/Fingers ( )
O. Other ( ) If Others Specify.

15. Any history of contact with positive case? YES ( ) NO ( ) 
16.if yes, what type of contact?

A. Home (Family Members/Roommate) ( )

B. Social (Friends/Public Place etc.) ( )

C. Duty (Workplace- Co-worker/Patient etc.) ( )

17.Opted for which type of isolation?
A. Home Isolation ( )
B. Institutional Isolation ( )
C. Hospital Admission ( )

18. If hospitalised, how many days admitted?:

19. Did any of your contacts test positive? YES ( ) NO ( )

20. How many close contacts did you have after coming positive?

21. How many of your contacts also tested positive, if any?

\section{HISTORY OF ANY PROPHYLAXIS}

22. Did you take any prophylaxis for covid-19? YES ( ) NO ( )

23. If yes, what did you take for prophylaxis?
A. Ivermectin ( )
B. Hydroxychloroquine ( )
C. Vitamin C ( )
D. Ayurvedic/Homeopathic Therapy ( )
E. Other ( ) If Others Specify

24. If Ivermectin, then dose?
A. $12 \mathrm{MG}(\mathrm{)}$
B. $15 \mathrm{MG}(\mathrm{)}$
C. $18 \mathrm{MG}($ )
D. $24 \mathrm{MG}(\mathrm{)}$

25. If Ivermectin, no. Of days ivermectin taken?

26. If Hydroxychloroquine, how much dosage?

27. If HCQ, no. Of days HCQ taken?

28. If Vitamin $\mathrm{C}$ taken, then dose?
A. $500 \mathrm{MG}$ OD ( )
B. $500 \mathrm{MG} \mathrm{BD} \mathrm{(} \mathrm{)}$

29. If vitamin- $\mathrm{C}$ taken, no. of days/duration vitamin- $\mathrm{C}$ taken?

30. If any other prophylaxis, please specify what?

31. If yes, please specify how much dose and type of preparation?

32. For how many days have you been taking the prophylaxis? 


\section{PHYSICAL ACTIVITY}

33. Physical activity: YES ( ) NO ( )

34. Type of physical activity?
A. Walking ( )
B. Jogging ( )
C. Yoga ( )
D. Gym ( )
E. Sports ( )
F. Other ( ) If Others Specify.

35. If yes, no. of days per week?.

36. If yes, no. of minutes/day?................ 
1. ฟฮ ๒ાจ ฟઘ๋:

2. ล।প:

3. ৪ঞֻ:

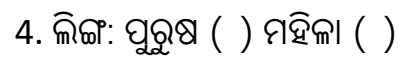

5. 6ต่ ( ) ตำ

6. घุจ1:
A. สิศต ( )
B. 6อฮิ6ชส ( )
C. ๒๐ธ์्त( )

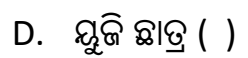

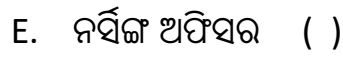

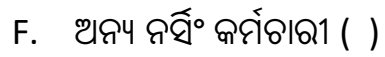
G. थाळू ( )

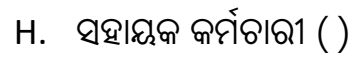
।. ขลЧЯ॥เด ( )

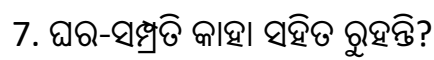

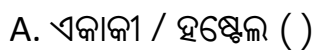
B. ฟดต घลิฉ।ด ( )

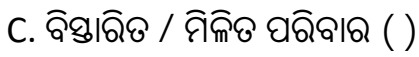
D. ब๕ / இЯ6Я८ ( )

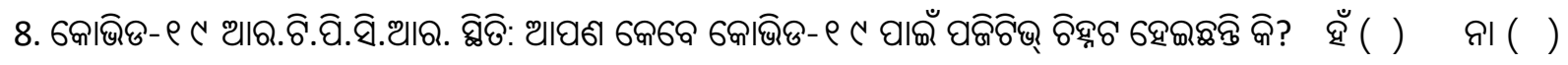

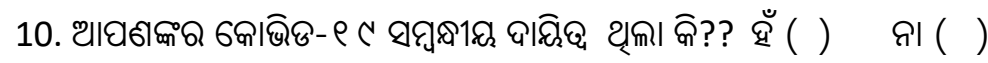

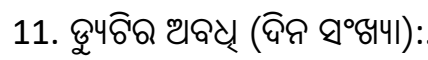

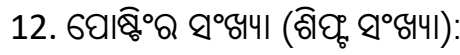

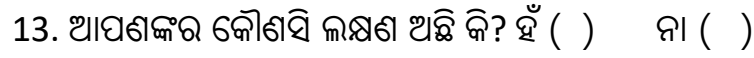

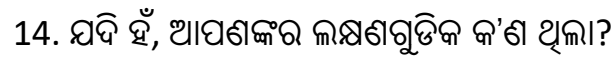
A. घ๐ ( )
B. થб్ర ( )
C. ตाठீ ()
D. ติ। ธ4ด|घ'( )

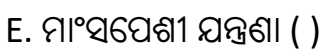
F. ช।ฉิิथा ( )
G. वูซิธิષ। ( )

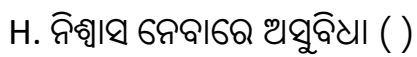

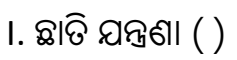

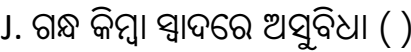




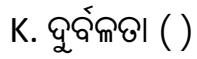
L. ৪ุ์ घ।ठ ( )

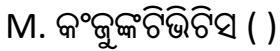

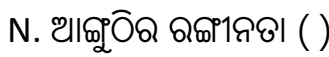

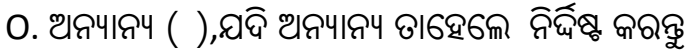

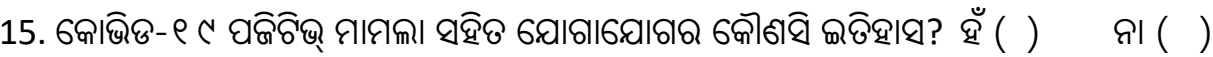

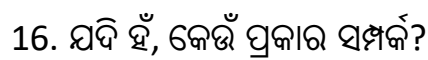

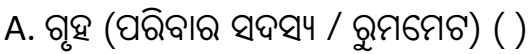

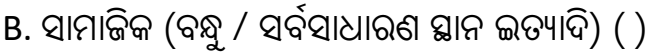

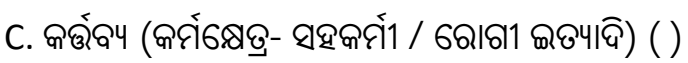

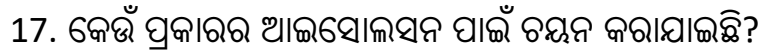

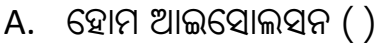

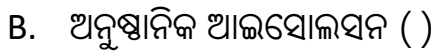

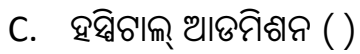

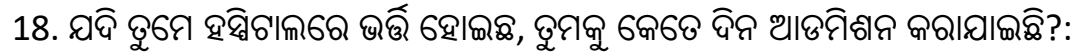

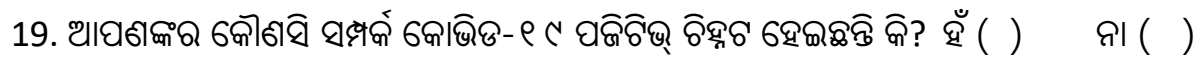

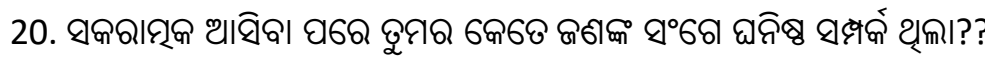

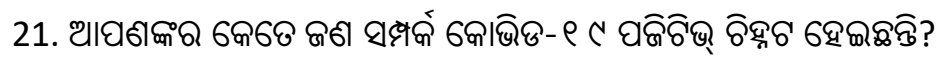

\section{HISTORY OF ANY PROPHYLAXIS}

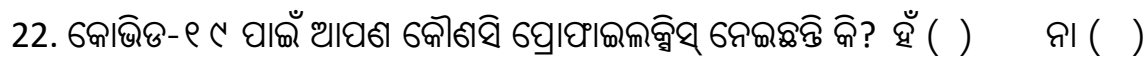

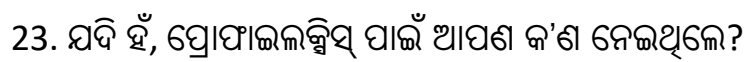

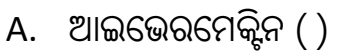

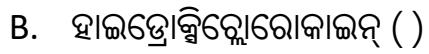
C. สิธเริจุ ฮิ ( )

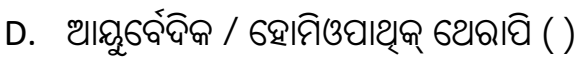

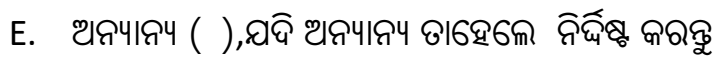

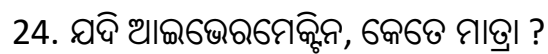
A. $12 \mathrm{MG}(\mathrm{)}$
B. $15 \mathrm{MG}(\mathrm{)}$
C. $18 \mathrm{MG}()$
D. $24 \mathrm{MG}(\mathrm{)}$

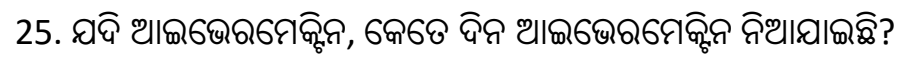

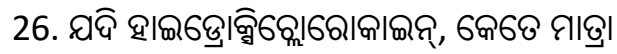

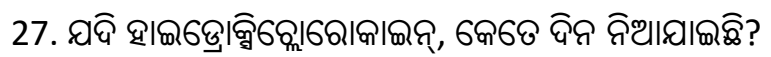

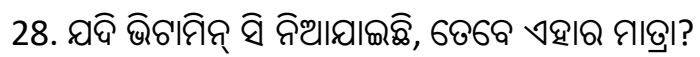
A. $500 \mathrm{MG} \mathrm{OD} \mathrm{(} \mathrm{)}$
B. $500 \mathrm{MG} \mathrm{BD}($ )

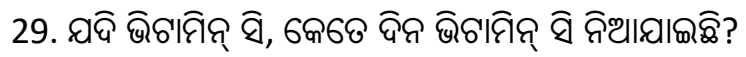




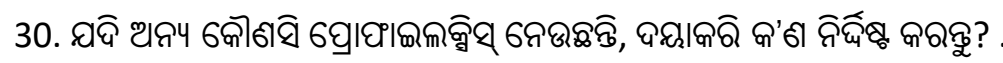

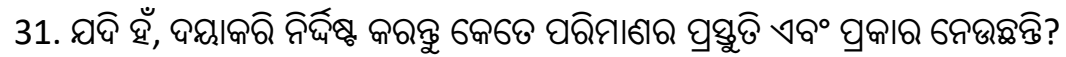

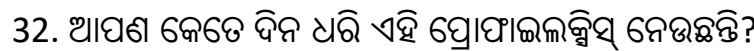

\section{PHYSICAL ACTIVITY}

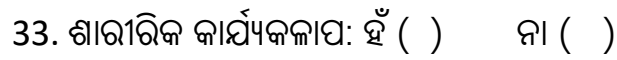

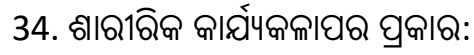
A. ช|กิด| ( )
B. ชธิ์ ( )
C. 6al| ()
D. ลิด् ( )
E. פ18। ( )

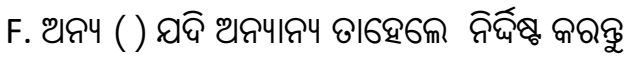

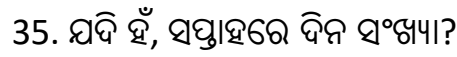

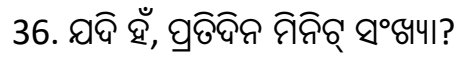

